Original Research Paper

\title{
Molecular-Biological Properties of the Attenuated Strain of Salmonella Abortus-Equi E-841, used in the Creation of a Vaccine against Abortion of Mares
}

\author{
${ }^{1}$ Assiya Mussayeva, ${ }^{1}$ Natalya Yegorova, ${ }^{2}$ Merey Yerishov, ${ }^{1}$ Aigul Dossanova, ${ }^{1}$ Vladislava Suchshikh, \\ ${ }^{1}$ Aidar Namet, ${ }^{3}$ Sarsenbek Siyabekov, ${ }^{3}$ Saltanat Nussupova, ${ }^{4}$ Bolat Yespembetov and ${ }^{4 * N a z y m ~ S y r y m ~}$ \\ ${ }^{1}$ Bacteriology Laboratory, Kazakh Scientific Research Veterinary Institute, Almaty, Kazakhstan \\ ${ }^{2}$ Department of Microbiology, Virology and Immunology, KazMUCE \\ "Kazakh Medical University of Continuing Education”, Almaty, Kazakhstan \\ ${ }^{3}$ Department of Clinical Veterinary Medicine, Kazakh National Agrarian Research University, Almaty, Kazakhstan \\ ${ }^{4}$ Laboratory of Microbiology, Research Institute for Biological Safety Problems, Gvardeiskiy, Kazakhstan
}

Article history

Received: 04-03-2021

Revised: 19-04-2021

Accepted: 26-04-2021

Corresponding Author:

Nazym Syrym

Department of Laboratory of

Microbiology, Research

Institute for Biological Safety

Problems, Gvardeiskiy,

Kazakhstan

Email: nazymsyrym53@ rambler.ru

\begin{abstract}
Salmonella abortion of mares takes a leading place in the in factious pathology of horses and leads to the abortion of pregnant mares and infected stallions become salmonella carriers. The disease with salmonella abortion manifests itself in the last months of pregnancy, maybe-during the mass foal of mares-in the spring. The article results obtained of studies on the biological properties and genetic characteristics of the attenuated strain of Salmonella abortus-equi E-841 compared with the control virulent strain of Salmonella Salmonella abortus-equi 7/1. These strains after inoculation on nutrient media retained their viability and had typical cultural-morphological, tinctorial and serological properties. When determining drug resistance, it showed resistance to rifampicin. As a result of studying the biochemical properties, it was found that the strain does not ferment urea, does not decompose gelatin and does not form indole, does not produce hydrogen sulfide. When determining the antigenic structure, it was shown that the attenuated strain is agglutinated in RA on glass with polyvalent and monoreceptor sera. The presence of mutations was determined by sequencing amplification fragments of the $r p o B$ and $r p s L$ genes. In the attenuated salmonella strain, mutations were found in the nucleotide sequences of fragments of the rpoB and rps $L$ genomes that cause antibiotic resistance and reduce the virulence of the strain, preventing the transformation of the strain into a virulent prototype of salmonella.
\end{abstract}

Keywords: Attenuated Strain, Mutation in the Genome, Nucleotide Sequence, Salmonella Abortion of Mares, Specific Prevention

\section{Introduction}

Salmonella abortion of mares is one of the most common infectious diseases of foaled mares, causing significant economic damage to the horse breeding industry of the republic, accompanied by premature birth (abortions) and the birth of an unviable fetus. For the first time, salmonella (Salmonella choleraesuis) was isolated from pig corpses in 1885 by American Scientists Salmon and Smith. Later, Smith and Kilborne in the United States discovered the causative agent of salmonella abortion of mares in 1893, in 1901 by V. D. Polyakov in Russia. Salmonellosis-a large group of zoonotic diseases, the family of enterobacteria, pathogenic to animals and humans, currently unites more than 2,300 serovars, divided into more than 60 gray-groups, most of which have independent names (Baker, 1970).

The causative agents of salmonellosis (in horsesSalmonella abortus equi) are small, straight, with rounded ends, gram-negative rods, spores and capsules do not form, facultative anaerobes (Brenner, 1986).

The first information on the epizootology of the disease in our country was published in the $1940 \mathrm{~s}$ and $1950 \mathrm{~s}$ in the western regions-the Guryev, Ural regions and in the south-west of the republic in the Kyzyl-Orda region (Biyashev et al., 1991; Biyashev, 1992).

The possibility of introducing the infection from the neighboring regions of Russia with Kazakhstan, due to the uncontrolled movement of horses, is not excluded. 
Currently, salmonellosis is widespread in many countries around the world, is most common in the Asian and African countries, while some cases are reported from European countries, the United States and Argentina (Bustos et al., 2016; Llorente et al., 2016; Ventsova and Safonov, 2021).

A high mortality rate caused by Salmonella abortus equi was recorded in Italy (Marenzoni, 2012). The abortions associated with viral and bacterial infections caused by the rhinovirus and the salmonella agents of equine abortions are most severe. Mixed infections are especially severe in the young horses and the mortality rate may reach $22 \%$. Salmonella carriage among the healthy animals is proven (Yurov, 2015).

Salmonella abortion is usually registered in areas of herd horse breeding. Salmonella carriers are often stallions. At the onset of a disease, the pathogen is transmitted from sick (aborted) horses and hidden carriers-stallions, work horses, foals. The pathogen can persist for a long time in the intestines, bile ducts, excreted in feces. In aborted mares, the pathogen is excreted in large quantities within 1-2 months with fetal fluids and membranes and vaginal discharge.

In the republic, the epizootic situation of Salmonella abortion of mares has worsened. In the Almaty region, abortions appeared in December 2016 and were registered until March 2017. When salmonellosis appears, abortions become widespread, it is almost impossible to stop them, up to $80 \%$ of mares in the herd are aborted. The infection is contagious and horses quickly become infected through food, bedding and grooming items. Outbreaks of infection were noted in many farms of Almaty, Zhambyl and East Kazakhstan regions. Abortions among mares were observed in the farms of Talgar, Karasai, Zhambyl and Enbekshikazakh districts of the region. In some horse breeding farms of the Almaty region, almost the entire offspring was lost (Mussaeva et al., 2016; Sultanov et al., 2015; Yegorova and Mussaeva, 2017).

Diagnostics of Salmonella abortion of mares is carried out on the basis of clinical-epizootic, postmortem data, as well as the results of bacteriological research. For bacteriological research during abortions in mares, pathological material (pieces of the liver, spleen, heart, lung and tubular bone) is often delivered in winter and spring.

Horses are affected by the causative agents of salmonellosis-Salmonella abortus-equi (serological group B, antigenic formula O-4.12; H-enx (phase 2) and Salmonella typhimurium (serological group $\mathrm{B}$, antigenic formula O-1, 4, 5, 12; H-i (1st phase,), 1, 2 (2nd phase)). Salmonella typhimurium often causes salmonella in animals, which poses a threat of salmonellosis in humans. Salmonellosis in humans subsequently becomes salmonella, which is difficult to cure.

The only and reliable way to combat abortions of mares of Salmonella etiology is Specific prevention vaccination of pregnant mares (at 4-7 months of pregnancyif a mare foals in April, then this coincides in terms from the beginning of September to the end of December). Tense immunity is formed and vaccinated mares will be $100 \%$ protected from abortions of Salmonella etiology.

Data on the study of the nucleotide sequences of the rpoB and rpsL gene fragments of Salmonella abortus-equi E-841 attenuated strain and the Salmonella abortus-equi 7/1 control strain in order to identify individual mutations that determine the residual virulence in the attenuated strain, which is used for the manufacture of the vaccine and the virulence in the control strain, which is used for biocontrol of the vaccine (Galin et al., 2020; Gross et al., 1998; Kalaeva et al., 2019; Maloy et al., 1996; Sulavic et al., 1997; Taira and Rhen, 1989; Tajbakhsh et al., 2011; Vorobyov et al., 2019).

Specific prevention of Salmonella abortion in mares is based on vaccination of pregnant mares.

The preservation of production and control strains of microorganisms used for the manufacture of vaccines in a genetically stable state is an urgent task. Preservation of the viability and biological activity of a culture for the longest possible time is a prerequisite for its use for the production of high quality biological preparations. Attenuated strains of microorganisms with desired properties are the basis for the production of live vaccines and determine their immunogenic activity and harmlessness. In this regard, special attention is paid to the problem of maintaining the viability of microorganisms. One of the most important tasks in the technological process of bio preparation production is the preservation of the viability of microorganisms-industrial strains, its biological properties in a stable initial state, preventing variability, reversion and aging of the culture. It is necessary to take into account the nature and stability of the strain, the cultivation conditions, the method and temperature of storage, reactivation, the frequency of reseeding and the procedure for working with the strain. When storing a dry live vaccine, the composition and quality of the protective environment, the concentration of microbial cells in the ampoule, the lyophilization mode, the presence of a vacuum, the duration and storage conditions are important. The immunogenic properties of a vaccine largely depend on the state of the initial matrix strain after storage; it is of great importance to include in the basis of the technological process a stage for preliminary verification of the correspondence of the biological properties of industrial strains to the passport characteristics.

Scientific research is aimed at identifying genetic differences between the vaccine and control strains of bacteria of Salmonella genus, belonging to the same serovar, conducting molecular-genetic studies of an attenuated vaccine Salmonella strain in a comparative aspect with a virulent control strain in order to obtain their genetic characteristics and differences in the nucleotide sequence of gene fragments, the stability of mutations in the nucleotide sequence of the rpoB and $r p s L$ genes, which determiner distance to two antibiotics responsible for reducing virulence and preventing reversion. 
The purpose of this work is to study the biological properties of the attenuated vaccine strain Salmonella abortus-equi E-841 and to determine their genetic characteristics.

\section{Materials and Methods}

Materials. In this experiment, we used: Salmonellosis strains, polyvalent and monoreceptor Agglutinating Sera, Bacterial media: Agar and Hottinger broth.

\section{Reagents}

Gis media with glucose, maltose, sorbitol, lactose, mannitol; glycerin, Glucose, gelatin, indole, the antibiotic Rifampicin. In total, more than 50 samples were used in the experiment.

\section{Methods}

The morphology of salmonella was studied by microcoping smears prepared from agar diurnal cultures, colored by gram and in a simple way. The cultural properties of the isolated cultures were determined by inoculating them on liquid, solid nutrient media. The biochemical properties of the cultures were determined by their ability to ferment carbohydrates in the His medium and the ratio of the isolated cultures to glycerin, gelatin, hydrogen sulfide and indole was also determined. The antigenic structure (O-and $\mathrm{H}$-antigens) of the studied salmonella strains was studied using polyvalent and monoreceptor agglutinating sera. The taxonomic distribution of cultures was carried out in accordance with the Bergey Manual on Systematic Bacteriology (Boone et al., 2001).

The antigenic structure of the tested salmonella strains was determined using polyvalent and monoreceptor agglutinating sera.

Antibiotic resistance of the salmonella vaccine strain was tested on Hottinger medium with $1.5 \%$ agar supplemented with rifampicin $(100 \mathrm{mcg} / \mathrm{ml})$ or streptomycin $(500 \mathrm{mcg} / \mathrm{ml})$. The presence of mutations was determined by sequencing amplification fragments of the $r p o B$ and $r p s L$ genes. Primers were selected for the gene regions where mutations occurred, optimal conditions for PCR were developed and specific fragments were sequenced.

To solve the problem of determining the genetic differences between the vaccine strain Salmonella abortus-equi E-841 and the control strain Salmonella abortus-equi 7/1, an approach was used to identify individual mutations that cause resistance of the vaccine strain to antibiotics, since the attenuated Salmonella abortus-equi E-841 was obtained by selection in a nutrient medium with a high concentration of antibiotics.

The amplification fragments were sequenced using cyclic sequencing using the Sanger method on an ABI Verity amplifier with the ABI PRISM Big Dye Terminator Cycle Sequencing Ready Reaction V.1 kit
(USA), followed by capillary electrophoresis analysis on an ABI 3500 Genetic Analyzer (Applied Bio systems).

\section{Results}

After inoculation on nutrient media, the attenuated and control strains of Salmonella abortus-equi retained their viability and biological activity and had typical culturalmorphological, tinctorial and serological properties in accordance with the passport data.

When determining drug resistance, the attenuated vaccine strain Salmonella abortus-equi E841 shows resistance to rifampicin at a final concentration of 100 $\mathrm{mcg} / \mathrm{ml}$, in contrast to the control strain Salmonella abortusequi 7/1. Rifampicin, competitively binds to RNA polymerase, preventing the binding of this enzyme to the final RNA molecules and thereby unblocking their synthesis.

These strains were also identified by studying the biochemical properties. As a result, the attenuated and control strains of salmonella do not ferment urea, do not decompose gelatin and do not form indole, do not produce hydrogen sulfide (the absence of hydrogen sulfide formation is a marker sign of Salmonella abortus-equi, which distinguishes it from other types of salmonella). Other members of the genus Salmonella form hydrogen sulfide in bismuth-sulfite agar. Salmonella strains give a positive reaction with methylene red (the medium is colored pink-red), a negative Voges-Proskauer reaction (yellow staining of the medium). Salmonella strains ferment glucose, galactose, mannose, arabinose, xylose, rhamnose, mannitol, maltose, dulcite, sorbitol, form hydrogen sulfide and reduce nitrates to nitrites. Salmonella grow on Simmons agar (assimilate citrateammonium salts). Both strains do not ferment lactose and sucrose, which is a marker of salmonella.

When determining the antigenic structure, it was shown that the attenuated strain agglutinated in RA on glass with polyvalent and monoreceptor sera O-IV $(++++)$, XII $(+++), \mathrm{H}-\operatorname{enx}(++)$. At the same time, there were negative results in the control.

The presence of mutations was determined by sequencing the amplification fragments of the $r p o B$ and rps $L$ genes. Primers were chosen to amplify the coding region of the rpoB, rpsL genes.

For the amplification of the $r p o B$ gene, primers specific for the Salmonella genus were used:

- 5 '-agcgtctgtctctggggg at -3 ' and

- 5 '-tcagaccgatgttcggac ct-3'

- Amplification of the $r p o B$ gene

The reaction mixture of $25 \mu \mathrm{L}$ contained $10 \mathrm{pM}$ of each primer and 10-50 ng of DNA. PCR was performed according to the following program: $95^{\circ} \mathrm{C}-1 \mathrm{~min}, 55^{\circ} \mathrm{C}$ $30 \mathrm{sec}, 72^{\circ} \mathrm{C}-1 \mathrm{~min}$; the number of cycles is 30 . 
The amplification fragments were sequenced using Sanger cyclic sequencing on an ABI PRISM Big Dye Terminator Cycle Sequencing Ready Reaction V.1 kit (USA) with subsequent analysis by capillary electrophoresis on an ABI 3500 Genetic Analyzer (Applied Biosystems).

The sequencing reaction was carried out according to the following program: $1 \mathrm{~min}$ at $96^{\circ} \mathrm{C}$ and then 25 cycles: $96^{\circ} \mathrm{C}-10 \mathrm{sec}, 55^{\circ} \mathrm{C}-5 \mathrm{sec} ., 60^{\circ} \mathrm{C}-4 \mathrm{~min}$ and storage at $4^{\circ}$ C. Same primers that were used in PCR were used as primers for sequencing.

Sequencing results for studying the nucleotide sequences of the $r p o B$ gene of Salmonella abortus-equi E841 vaccine strain and Salmonella abortus-equi 7/1 control strain are shown in Figs. 1 and 2.

Figures 1 and 2 show sequenograms in the form of peaks and letter sequences.

Figure 1 shows the amino acid codons in the fragments of the rpoB gene of Salmonella abortus-equi E- 841 vaccine strain, in 521 of which a mutation occurred, leading to the replacement of nucleotide $\mathrm{C}$ by $\mathrm{T}$ (as a result of which the triplet of nucleotides TCT becomes TTT), which entails the replacement of the amino acid Ser - in 521 amino acid codon with Phe. Amino acid codons in which mutations have occurred are underlined; an arrow indicates the position of the mutant nucleotide.

Figure 2 shows that in the gene fragments of the $r p o B$ from Salmonella abortus-equi7/1 control strain mutations were not found (the sequence of the triplet of TCT nucleotides does not change, remains Ser- at 521 amino acid codon), that is, the nucleotide sequence of the tested strain is identical to the sequence of Salmonella typhimurium published in the Gene Bank database.

Comparison of nucleotide sequences of $r p o B$ gene fragments of Salmonella abortus-equi E-841 and Salmonella abortus-equi7/1 strains showed the presence of point mutations in the vaccine strain. In the rpoB gene from Salmonella abortus-equi E-841 vaccine strain a substitution of $\mathrm{C}$ for $\mathrm{T}$ at 521 amino acid codon was found, which leads to the replacement of the Sera mino acid with Phe.

Amplification of the rpsL gene. Salmonella abortusequi $E$-841vaccine strain shows resistance to streptomycin at a final concentration of $500 \mu \mathrm{g} / \mathrm{ml}$.

Streptomycin exhibits antimicrobial effect, binding to proteins of the small subunit of ribosomes and disrupting the process of synthesis of bacterial proteins, resistance to streptomycin is most often due to mutations in the protein S12. Genomic DNA was isolated from Salmonella abortus-equi E-841 strain, which was used to detect mutations in the rps $L$ geneen coding the ribosomal protein $S 12$ with a size of 124 aa. To amplify the coding region of the gene, primers specific for the Salmonella genus were selected based on the rpsL Salmonella typhimurium LT 2 gene sequence published in the Gene Bank. Amplification of the rpsL gene. To amplify the rpsL gene, primers specific for the Salmonella genus were used with the following composition:
- 5 '-cgtcct cat att gtg tgaggg -3 ' and

- 5 '-gcatggaaa tac tccgttgtt $-3^{\prime}$

The reaction mixture of $25 \mu \mathrm{L}$ contained $10 \mathrm{pM}$ of each primer and 10-50 ng DNA. PCR was performed according to the following program: $95^{\circ} \mathrm{C}-1 \mathrm{~min}, 55^{\circ} \mathrm{C}$ $30 \mathrm{sec}, 72^{\circ} \mathrm{C}-1 \mathrm{~min}$; the number of cycles is 30 .

Fragments of rpsL genes of Salmonella abortus-equi E- 841 vaccine and Salmonella abortus-equi7/lcontrol strains were then sequenced. The amplification fragments were sequenced using Sanger cyclic sequencing on an ABI PRISM Big Dye Terminator Cycle Sequencing Ready Reaction V.1 kit (USA) with subsequent analysis by capillary electrophoresis on an ABI 3500 Genetic Analyzer (Applied Biosystems).

The sequencing reaction was carried out according to the following program: $1 \mathrm{~min}$ at $96^{\circ} \mathrm{C}$ and then 25 cycles: $96^{\circ} \mathrm{C}-10 \mathrm{sec}, 55^{\circ} \mathrm{C}-5 \mathrm{sec} ., 60^{\circ} \mathrm{C}-4 \mathrm{~min}$ and storage at $4^{\circ} \mathrm{C}$. Same primers that were used in PCR were used as primers for sequencing.

When comparing the sequenced nucleotide sequences of the rps $L$ gene in the vaccine strain, mutations in the 42 amino acid codon were found.

In rps $L$ gene from the Salmonella abortus-equi E- 841 substitution of nucleotide $\mathrm{A}$ for $\mathrm{C}$ results in the replacement of the amino acid Lys with Gln in the codon.

Sequencing results to study the nucleotide sequences of rpsLgene from Salmonella abortus-equi E- 841 vaccine and Salmonella abortus-equi 7/1 control strains are shown in Figs. 3 and 4.

Figures 3 and 4 show sequenograms in the form of peaks and letter sequences.

Figure 3 shows the amino acid codons in the fragments of the rpsL gene of Salmonella abortus-equi E-841 salmonella vaccine strain, in one of which a mutation occurred, leading to the replacement of nucleotide A with $\mathrm{C}$ (as a result of which the triplet nucleotides AAA becomes CAA), which leads to the replacement of the Lys amino acid with $G l n$ in the 42 amino acid codon. Amino acid codons in which mutations have occurred are underlined; the position of the mutant nucleotide is indicated by an arrow.

In the Salmonella abortus-equi7/lcontrol strain, the sequence AAA is found in the 42 amino acid codon of the rpsL gene, which corresponds to the Lys amino acid, which is also present in the rps $L$ of Salmonella typhimurium LT2 gene sequence.

Figure 4 shows that no mutations were found in the rpsL gene fragments of the Salmonella abortus-equi $7 / 1$ control strain (these quence of the AAA nucleon tiderip let does not change, $L$ ysremains in the 42 amino acid codon), that is, its nucleoti de sequences identical to that of Salmonella Typhimurium, published in the (GeneBank, 2021). 


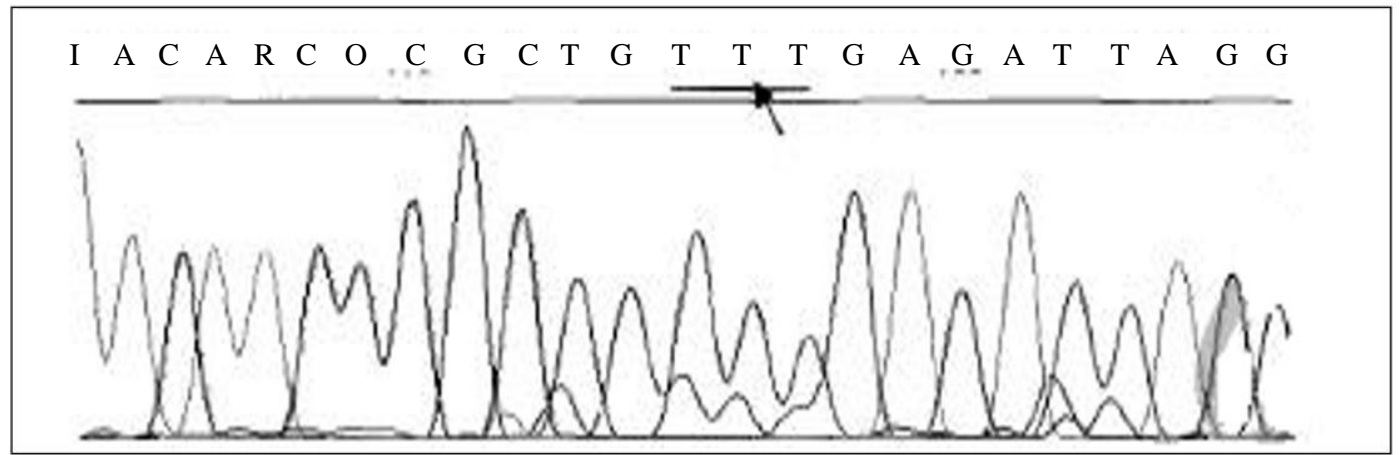

Fig. 1: Sequenogram fragments of nucleotide sequences of rpoB gene from Salmonella abortus-equi E-841 strain: Ser-521 on Phe(TCT on TTT)

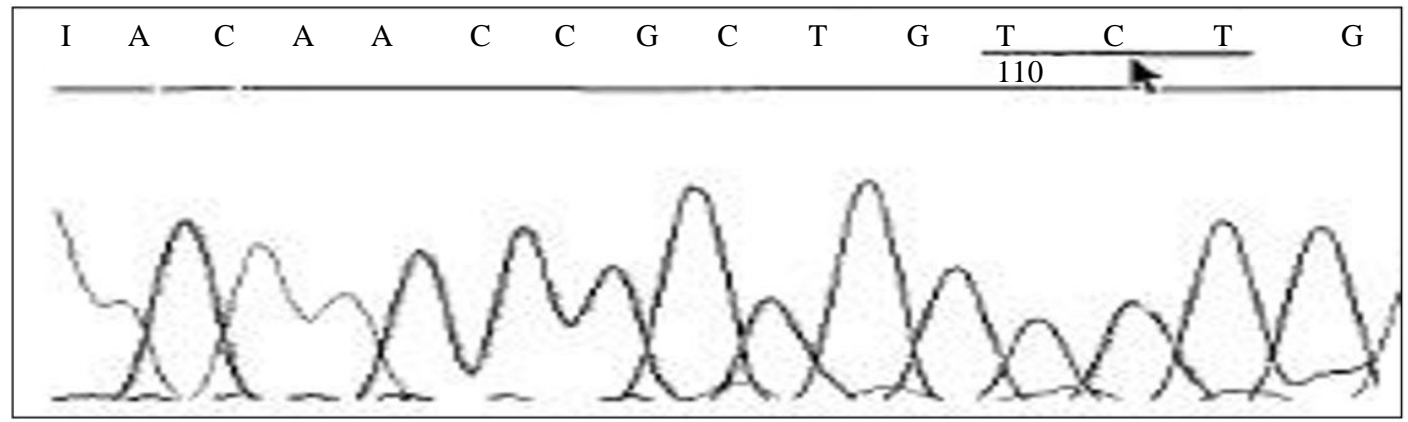

Fig. 2: Sequenogram fragments of the nucleotide sequences of rpoB gene of strain Salmonella abortus-equi 7/1:Ser-521 (TCT)

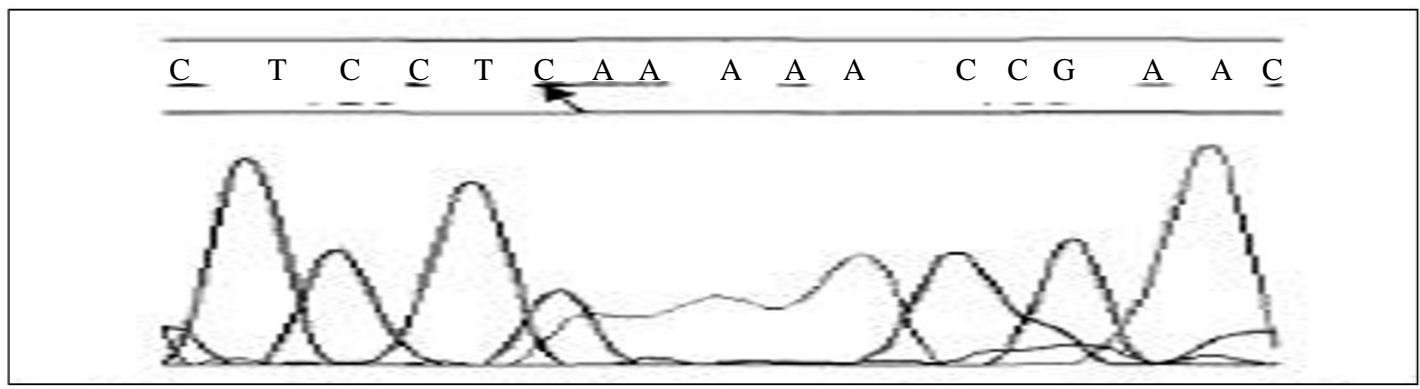

Fig. 3: Sequenogram fragments of nucleotide sequences of the rpsL gene from the strain Salmonella abortus-equi E-841:Lys42 on $\operatorname{Gln}(\mathrm{AAA}$ on CAA)

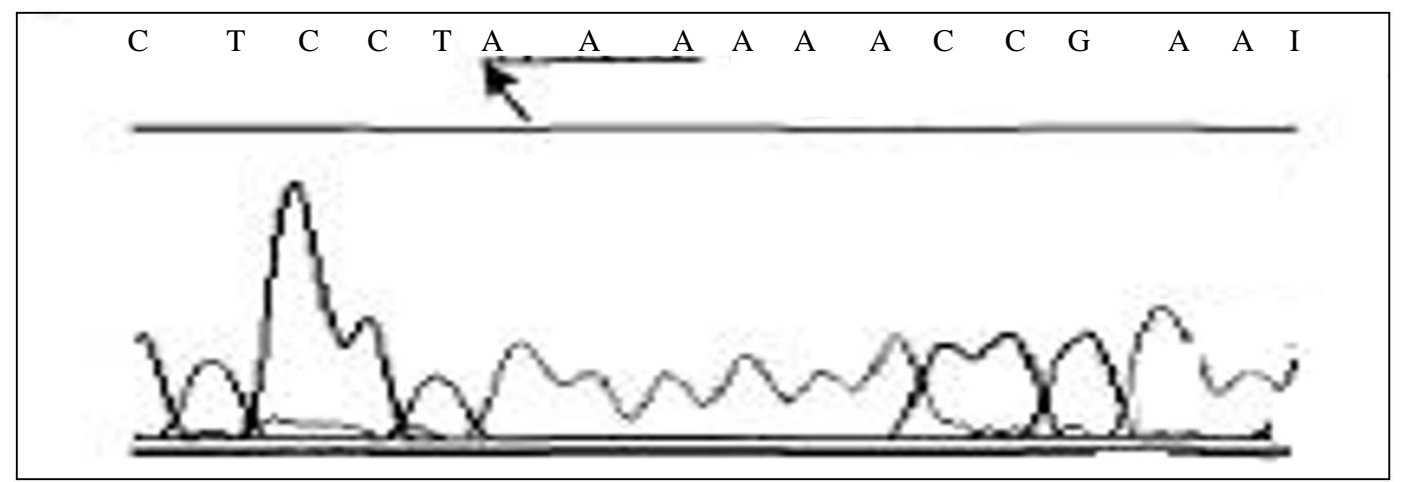

Fig. 4: Sequenogram of nucleotide sequences fragments of the rpsL gene of the Salmonella abortus-equi 7/1 strain: Lys-42 (AAA) 


\section{Discussion}

As a result of genetic studies, mutations in the nucleotide sequences of the DNA of the attenuated strain were found, causing a decrease in the virulence of the strain. In the Salmonella abortus-equi E-841 attenuated strain, which is resistant to rifampicin, a fragment of the $r p o B$ gene was identified, in which a mutation occurred due to the replacement of nucleotide $\mathrm{C}$ with $\mathrm{T}$ (as a result of which the triplet TCT became TTT), which entails the replacement of Ser-amino acid to Phe at 521 amino acid codon. When comparing the nucleotide sequence of the rps $L$ gene of the tested strains in the vaccine strain, a mutation was found in the amino acid codon; in the Salmonella abortus-equi E-841 vaccine strain, which is resistant to streptomycin, the point mutation is due to the replacement of nucleotide $\mathrm{A}$ by $\mathrm{C}$ (as a result of which the AAA triplet became CAA), which entails the substitution of Lys-amino acid for Gln in the 42 amino acid codon.

In the Salmonella abortus-equi 7/1 control strain in the region of the $r p o B$ and $r p s L$ genes, no mutations were found; therefore, there is no decrease in virulence and antibiotic resistance. The control strain is used for biocontrol in the production of a vaccine.

During the production of a attenuated against Salmonella abortion in mares, as a result of genetic studies, mutations in the nucleotide sequences of genome fragments of the vaccine strain were found, causing the emergence of antibiotic resistance and a decrease in the virulence of the strain, preventing the reversion of the strain.

\section{Conclusion}

The vaccine strain intended for the production of a vaccine against Salmonella abortion in mares was obtained by stepwise selection of clones grown on media with antibiotics and is a freeze-dried Salmonella culture. The vaccine strain was obtained from an epizootic virulent culture of Salmonella abortus-equi by attenuation in 2 genes $(r p o B$ and $r p s L)$. Its virulence is reduced 20 times compared to the natural prototype. The strain is resistant to neomycin and streptomycin, has two markers that ensure antibiotic resistance and retain reversion.

\section{Author's Contributions}

Assiya Mussayeva: Methodology, validation, formal analysis, writing-Original draft preparation.

Natalya Yegorova: Conceptualization, validation, resources, writing-original draft preparation.

Merey Yerishov: Methodology, validation, investigation, data curation.

Aigul Dossanova: Conceptualization, validation, resources, data curation.

Vladislava Suchshikh: Conceptualization, validation, formal analysis, visualization.
Aidar Namet: Methodology, software, validation, investigation.

Sarsenbek Siyabekov: Conceptualization, validation, data curation, visualization.

Saltanat Nussupova: Software, validation, resources, writing-review and editing.

Bolat Yespembetov: Software, validation, formal analysis, writing-review and editing.

Nazym Syrym: Methodology, validation, investigation, visualization.

\section{Ethics}

The design of the article was carried out in compliance with the principles of scientific ethics, including maintaining high standards of intellectual integrity and preventing the fabrication of scientific data, falsification, plagiarism, false co-authorship, use by individual participants of collective research, data and conclusions obtained in research, without the consent of other participants, taking into account the declaration on ethical principles of scientific activity of the Antiparliamentary Assembly of the Commonwealth of Independent States.

\section{References}

Baker, J.R., 1970. Salmonellosis in the horse. British Veterinary Journal, 126, 100-105. https://www.cabdirect.org/cabdirect/abstract/1970 2203776

Biyashev, K. B. (1992). Immunoprophylaxis of salmonellosis of farm animals in Kazakhstan: dissertation ... doctors of Veterinary Sciences: 16.00.03. Alma-Ata, pp; 464. https://search.rsl.ru/ru/record/01007914963

Biyashev, K. B., Omarov, M., \& Tolysbaev, B. T. (1991). Salmonellosis of animals and control measures. Methodological recommendations. Alma-Ata, pp; 42.

Boone, D. R., Castenholz, R. W., \& Garrity, G. M. (2001). Bergey's Manual ${ }^{\circledR}$ of Systematic Bacteriology. Springer. https://www.springer.com/gp/book/9780387987712

Brenner, D. J., (1986). Facultatively anaerobic gramnegative rods. In: Bergey's manual of systematic bacteriology, Williams \& Wilkins, pp; 408-516. https://ci.nii.ac.jp/naid/10029544330/

Bustos, C. P., Gallardo, J., Retamar, G., Lanza, N. S., Falzoni, E., Caffer, M. I., ... \& Guida, N. (2016). Salmonella enterica serovar Abortusequi as an emergent pathogen causing equine abortion in Argentine. Journal Equine Veterinary Science, 39, S58-59.

Galin, P., Galyaveeva, A., Bataev, H. \& Safonov, V. (2020). The role of micronutrients and vitamins in the prevention and remote treatment of heart failure. Revista Latinoamericana de Hipertension, 15(1); 26-32. 
GeneBank. (2021). Database. https://www.ncbi.nlm.nih.gov/genbank

Gross, U., H. Tshape, I. Bendarek and M. Frosch, 1998. Antibiotic resistance in Salmonella enterica serotype Typhimurium. European Journal of Clinical Microbiology and Infectious Diseases, 17: 385-387. https://doi.org/10.1007/BF01691565

Kalaeva, E., Kalaev, V., Efimova, K., Chernitskiy, A., \& Safonov, V. (2019). Protein metabolic changes and nucleolus organizer regions activity in the lymphocytes of neonatal calves during the development of respiratory diseases. Veterinary World, 12(10); 1657. https://www.ncbi.nlm.nih.gov/pmc/articles/PMC6 $868248 /$

Llorente, L. A., Ivanissevich, A., Camina, S., Marco, L., Vissani, A., Olguin, C., Herrera, M., \& Barrandeguy, M. (2016). Occurrence of multiple abortions due to Salmonella enterica serovar Abortusequi infection. Journal of Equine Veterinary Science, 39: 58.

Maloy, S. R., Stewart, V. J., \& Taylor, R. K. (1996). Genetic analysis of pathogenic bacteria. Cold Spring Harbor Laboratory Press.

Marenzoni, M. L., (2012). Causes of equine abortion, stillbirth and neonatal death in central Italy. Veterinary Record, 170: 262. https://doi.org/10.1136/vr.100551.

Mussaeva, A K., Yegorova, N. N., \& Daugalieva, A. T. (2016). Genetic features of the vaccine strain Salmonella dublin15s. Bulletin of Science of the S.Seifullin Kazakh Agro Technical University, 1: 1-11. https://kazatu.edu.kz/assets/i/science/vn16svagro26.pdf

Sulavic, M. C., Dazer, M., \& Miller, P. F. (1997). The Salmonella Typhimurium mar locus: Molecular and genetics analyses and Assessment of its role in virulence. Journal of Bacteriology, 179: 1857-1866. https://doi.org/10.1128/jb.179.6.1857-1866.1997
Sultanov, A. A., Mussaeva, A. K., Yegorova, N. N., \& Dosanova, A. K. (2015). Diagnostics and prophylactics of Salmollesis abortion of mares. International Journal of Applied Sciences and Fundamental Research, 12; 1883-1887. https://applied-research.ru/pdf/2015/12-10/8402.pdf

Taira, S., \& Rhen, M. (1989). Molecular organization of genes constituting the virulence determinant on the Salmonella typhimurium 96 kilobase pair plasmid. FEBS Letters, 257: 274-278. https://doi.org/10.1016/0014-5793(89)81551-0

Tajbakhsh, M., Nayer, B. N., Motavaze, K., Kharaziha, P., Chiani, M., Zali, M. R., \& Klena, J. D. (2011). Phylogenetic relationship of Salmonella enterica strains in Tehran, Iran, using 16S rRNA and gyrB gene sequences. Journal of Infection in Developing Countries, 5(6); 465-472. https://doi.org/10.3855/jidc.1504

Ventsova, I., \& Safonov, V. (2021). The role of oxidative stress during pregnancy on obstetric pathology development in high-yielding dairy cows. American Journal of Animal and Veterinary Sciences, $\quad 16(1) ;$ 7-14. https://thescipub.com/abstract/ajavsp.2021.7.14

Vorobyov, V., Vorobyov, D., Polkovnichenko, P., \& Safonov, V. (2019). Evaluation of hematological and metabolic parameters in small ruminants with trace elements deficiency under different biogeochemical conditions. Worlds Veterinary Journal, 9(4); 311-316. https://doi.org/10.36380/scil.2019.wvj39

Yegorova, N. N., \& Mussaeva, A. K. (2017). Vaccination against Salmonellosis abortion of mares. Izvestia of the National Academy of Sciences of the Republic of Kazakhstan. Series of Agricultural Sciences, 2; 240-246. http://nblib.library.kz/elib/library.kz/jurnal/Agrar naya\%2002-2017/Yegorova0217.pdf

Yurov, K. P. (2015). Mass infectious abortions in horses. Modern Veterinary Medicine, 3: 35-39. 